\title{
RBFOX3 Regulates the Chemosensitivity of Cancer Cells to 5-Fluorouracil via the PI3K/AKT, EMT and Cytochrome-C/Caspase Pathways
}

\author{
Tianze Liua, ${ }^{a}$ Xiaojun Wu Yizhuo Li $^{a}$ Wenjing Lu ${ }^{\mathrm{b}}$ Fufu Zheng ${ }^{\mathrm{d}}$ \\ Changlin Zhang $^{\mathrm{a}}$ Qian Long ${ }^{\mathrm{a}}$ Huijuan Qiu ${ }^{\mathrm{a}}$ Yixin Li $^{\mathrm{a}}$ Qin Ge ${ }^{\mathrm{a}}$ Miao Chen $^{\mathrm{a}}$ \\ Xinfa Yu ${ }^{c}$ Wangbing Chen ${ }^{e}$ Hongyang Zhang ${ }^{f}$ Wenlin Huanga,g Meihua Luo ${ }^{c}$ \\ Wuguo Deng ${ }^{a}$ Liren Li ${ }^{a}$
}

aSun Yat-sen University Cancer Center, State Key Laboratory of Oncology in South China, Collaborative

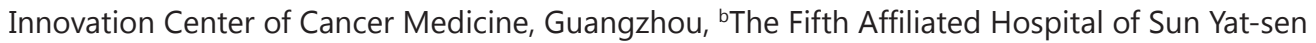
University, Zhuhai, 'Shunde Hospital of Southern Medical University, Foshan, ${ }^{\mathrm{d} T h e}$ First Affiliated Hospital of Sun Yat-sen University, Guangzhou, ${ }^{e}$ Cancer Center Union Hospital, Tongji Medical College, Huazhong University of Science and Technology, Wuhan, ${ }^{f}$ Department of Anatomy and Neurobiology, Zhongshan School of Medicine, Sun Yat-sen University, Guangzhou, "9State Key Laboratory of Targeted Drug for Tumors of Guangdong Province, Guangzhou Double Bioproduct Inc., Guangzhou, China

\section{Key Words}

Rbfox3 • 5-fluorouracil (5-FU) • Hepatocellular carcinoma (HCC)

\begin{abstract}
Background/Aims: RBFOX3, an RNA-binding fox protein, plays an important role in the differentiation of neuronal development, but its role in the chemosensitivity of hepatocellular carcinoma (HCC) to 5-FU is unknown. Methods: In this study, we examined the biological functions of RBFOX3 and its effect on the chemosensitivity of HCC cells to 5-FU in vitro and in a mouse xenograft model. Results: RBFOX3 was found to have elevated expression in HCC cell lines and tissue samples, and its knockdown inhibited HCC cell proliferation. Moreover, knockdown of RBFOX3 improved the inhibitory effect of 5-fluorouracil (5-FU) on cell proliferation, migration and invasion, and enhanced the apoptosis induced by 5-FU. However, overexpression of RBFOX3 reduced the inhibitory effect of 5-fluorouracil (5-FU) on cell proliferation, migration and invasion, and decreased the apoptosis induced by 5-FU. We further elucidated that RBFOX3 knockdown synergized with 5-FU to inhibit the growth and invasion of HCC cells through PI3K/AKT and epithelial-mesenchymal transition (EMT) signaling, and promote apoptosis by activating the cytochrome-c/caspase signaling pathway. Finally, we validated that RBFOX3 regulated 5-FU-mediated cytotoxicity in HCC in mouse xenograft models. Conclusions: The findings from this study indicate that RBFOX3 regulates the chemosensitivity of HCC to 5-FU in vitro and in vivo. Therefore, targeting RBFOX3 may

T. Liu, X. Wu, Y. Li and W. Lu contributed equally to this work.

Liren Li, Wuguo Deng and Meihua Luo

Sun Yat-sen University Cancer Center, Guangzhou; Shunde Hospital of Southern Medical University, Foshan (China)

E-Mail lilr@sysucc.org.cn, dengwg@sysucc.org.cn, luomeihua888@163.com
\end{abstract}




\section{Cellular Physiology Cell Physiol Biochem 2018;46:1365-1380

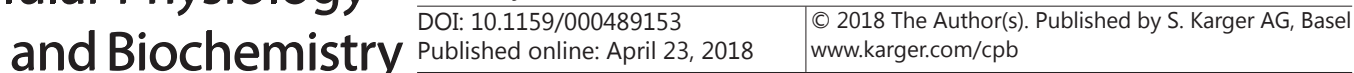 \\ Liu et al.: RBFOX3 Regulates the Chemosensitivity}

improve the inhibition of $\mathrm{HCC}$ growth and progression by $5-\mathrm{FU}$, and provide a novel potential therapeutic strategy for HCC.

\section{Introduction}

(C) 2018 The Author(s)

Published by S. Karger AG, Basel

Liver cancer is the sixth most prevalent cancer and the second most common cause of cancer death worldwide [1,2]. Hepatocellular carcinoma (HCC) is the major histological subtype of liver cancer. Because of its high mortality, HCC has been regarded as the leading cause of cancer deaths worldwide [3,4]. The traditional treatments for HCC include local ablation, surgical resection, chemotherapy, and liver transplantation. In recent years, molecular targeted agents such as sorafenib that targets the signaling cascades involved in HCC development and progression have been explored [5-7]. However, the long-term prognosis of HCC patients is still poor [8]. Therefore, there is an urgent need to determine the molecular mechanisms of HCC progression and identify novel targets for the treatment of HCC.

As the most widely used chemotherapeutic drug in liver cancer treatment, 5-Fluorouracil (5-FU) primarily disturbs the synthesis of the pyrimidine thymidine, a nucleoside required for DNA replication, by blocking the activity of thymidylate synthase, and thus induces cell cycle arrest and apoptosis in cancer cells [9-11]. Although adjuvant 5-FU treatment has yielded a good effective rate in some HCC patients, low chemosensitivity and acquired drug-resistance still cause treatment failure in the majority of patients $[12,13]$. Moreover, the precise mechanism of the low chemosensitivity of HCC to 5-FU is not well understood. Therefore, it is urgent to discover novel targets that regulate the chemosensitivity of HCC to 5-FU.

RBFOX3 (also known as Fox-3, Fox1 homolog C) is a new member of the RBFOX3 family of splicing factors. In human, RBFOX3 is a protein-coding gene located on chromosome 17 and comprised of 15 exons [14]. As the third member of the RNA-binding fox family of proteins, RBFOX3 plays a role in alternative pre-mRNA splicing via binding to the conserved UGCAUG element in the upstream intron [15]. Although most studies have focused on the function of RBFOX3 in splicing regulation, its roles may not be limited to splicing. One study suggested that RBFOX3 might be a transcriptional regulator as it could bind to DNA [16]. Our previous study found that RBFOX3 promoted HCC growth and progression by activating the hTERT signaling [17]. Another study showed that RBFOX3 bound directly to pri-miRNAs and regulated the recruitment of the microprocessor complex to pri-miRNAs [18], which suggested that RBFOX3 possessed a novel function for controlling the biogenesis of a subset of microRNAs. In this study, we have found that RBFOX3 was highly expressed in HCC cell lines and tissue samples, and RBFOX3 knockdown enhanced the inhibition of cell proliferation, migration and invasion mediated by 5 -FU both in vitro and in vivo.

\section{Materials and Methods}

Drugs and antibodies

5-Fluorouracil (99.9\% purity) was purchased from Selleck Chemicals (Houston, TX), dissolved in DMSO (20mol/L), and stored at $-20^{\circ} \mathrm{C}$ The stock was diluted with culture medium before use. All antibodies were purchased from Cell Signaling.

Cell lines

Human hepatocellular carcinoma cells (SNU-449, Hep3B, Bel-7402, SNU-387, and HepG2) and human immortalized hepatic cell line MIHA were obtained from the American Type Culture Collection (ATCC, Manassas, VA). All cell lines were cultured in Dulbecco's Modified Eagle Medium (Invitrogen, Carlsbad, CA) supplemented with $10 \%$ fetal bovine serum, penicillin $(100 \mathrm{IU} / \mathrm{mL})$, and streptomycin $(100 \mu \mathrm{g} / \mathrm{mL})$ at $37^{\circ} \mathrm{C}$ in a humidified atmosphere with 5\% $\mathrm{CO} 2$. 


\section{Cellular Physiology Cell Physiol Biochem 2018;46:1365-1380 \begin{tabular}{ll|l} 
and Biochemistry Published online: April 23, 2018 & $\begin{array}{l}\text { (c) 2018 The Author(s). Published by S. Karger AG, Basel } \\
\text { www.karger.com/cpb }\end{array}$ \\
\hline
\end{tabular}}

Liu et al.: RBFOX3 Regulates the Chemosensitivity

\section{Transient transfection of HCC cells}

To inhibit RBFOX3 expression, cells were transfected with RBFOX3-specific siRNA oligonucleotide (100 nmol/L) 5'-GGC UAC ACG UCU CCA ACA UTT-3' (siRB-1) or 5'-GCG GCA AAU GUU CGG GCA AUU TT-3' (siRB2), or the nonspecific siRNA 5'-UUC UCC GAA CGU GUC ACG UTT-3' (siNC). 48 hours post transfection, the interference effect was examined by western blot. The siRNAs were purchased from Shanghai GenePharma Co. (Shanghai, China).

\section{Western blot analysis}

HCC cell lysates were prepared. Equal amounts of protein $(30 \mu \mathrm{g})$ from each sample were resolved by $10 \%$ or $12 \%$ sodium dodecyl sulfate-polyacrylamide minigel (SDS-PAGE), electrophoretically transferred to a PVDF membrane, and the blots were probed with specific antibodies. The protein signals were visualized by an enhanced chemiluminescence reaction system as recommended by the manufacturer, and quantified using ImageQuant software. GAPDH served as the loading control.

\section{Lentiviral Construction and Cell Transfection}

The U6-sh-RBFOX3-EGFP-IRES-puromycin vectors were obtained from Genechem Company Ltd (Shanghai, China) to knock down RBFOX3. A lentiviral vector containing non-silencing short hairpin RNA was used as the negative control. HCC cells were infected with either the lentiviral vectors encoding RBFOX3specific short hairpin RNA sequences or the negative control vector. Stable cell lines were selected using 1-2 $\mu \mathrm{g} / \mathrm{mL}$ puromycin for 2 weeks. The efficiency of RNA interference was determined by western blot. All assays were independently performed in triplicates.

\section{Cell viability assay}

Cells $\left(2 \times 10^{2}\right.$ cells per well) were seeded in 96-well plates and exposed to serial dilutions of 5-FU, siRNA or both for indicated time, and the viability of the cells was assessed by MTS assay (Promega, WI). Growth inhibition was presented as the percentage of surviving cells in treated versus control cells. The IC50 value was the concentration leading to $50 \%$ cell growth inhibition compared with untreated control. All assays were independently performed in triplicates.

\section{Colony formation assay}

Cells were plated in 6-well culture plates at $5 \times 10^{2}$ cells per well as single cells and treated with 5 -FU, siRNA or both at the indicated concentrations. After incubation for another 14 days, cells were washed twice with phosphate buffered saline. Colonies were fixed with paraformaldehyde for 30 minutes and stained with crystal violet for 15 minutes. All assays were independently performed in triplicates.

\section{Wound healing assay}

Approximately $3 \times 10^{5}$ cells were plated in each well of a 6 -well plate. After overnight incubation, cells were either untreated or treated with 5-FU, siRNA or both at the indicated concentrations. When cell confluence reached 90-100 \% after treatment, wounds were made using a $200 \mu$ l pipette tip. The cells were rinsed several times with PBS to remove any free-floating cells and debris before growth medium was added back. Wound gap was observed and cells were photographed. All assays were independently performed in triplicates.

\section{Transwell invasion assay}

Matrigel invasion assays were performed using a 24-well cell culture insert and BD Matrigel (BD Biocoat, Bedford, MA). The transwell membrane was coated with $30 \mu \mathrm{L}$ diluted Matrigel. After overnight incubation, $5 \times 10^{4}$ cells suspended in $200 \mu \mathrm{L}$ medium without FBS were added to the upper chamber with matrix gel, whereas $500 \mu \mathrm{L}$ medium with $30 \%$ FBS was added to the lower chamber. After 24 hours, the cells that had not migrated through the pores were manually removed from the upper face of the filters with cotton swabs, and the cells adhered to the bottom surface of the inserts were fixed in paraformaldehyde and stained with crystal violet. Finally, the filters were washed thoroughly in water and images were taken under a microscope with appropriate magnification. All assays were independently performed in triplicates.

\section{Apoptosis analysis}

Apoptosis in HCC cells untreated or treated with 5-FU, siRNA or both was measured by AnnexinV and propidium iodide (KeyGEN, Nanjing, China) staining based fluorescence-activated cell sorting (FACS). In brief, cells with RBFOX3 stable knockdown were plated $3 \times 10^{5}$ cells per well in 6 -wellplates, digested 


\section{Cellular Physiology Cell Physiol Biochem 2018;46:1365-1380 \begin{tabular}{l|l|} 
and Biochemistry Published 10.1159/000489153 & $\begin{array}{l}\text { C } 2018 \text { The Author(s). Published by S. Karger AG, Basel } \\
\text { www.karger.com/cpb }\end{array}$
\end{tabular} \\ Liu et al.: RBFOX3 Regulates the Chemosensitivity}

with $0.25 \%$ trypsin-no EDTA (Gibco BRL, Grand Island, NY), washed twice with cold PBS and subsequently stained with Annexin V/propidium iodide before being analyzed by flow cytometry (Beckman Coulter, CA) according to manufacturer's instructions. All assays were independently performed in triplicates.

\section{Animal study}

To investigate the effect of RBFOX3 inhibition with or without 5-FU on HCC cell growth and metastasis in vivo, six-week old BALB/c nude mice from the Beijing Vital River Laboratory Animal Technology Co., Ltd. (Beijing, China) were maintained in a specific pathogen-free environment. All animal procedures were performed in accordance with the Guide for the Care and Use of Laboratory Animals (NIH publications Nos. 80-23, revised 1996) and the Institutional Ethical Guidelines for Animal Experiments developed by Sun Yatsen University. For the xenograft tumor model, Hep3B cells $\left(3 \times 10^{6}\right)$ infected with either the lentiviral vectors encoding specific short hairpin RNA sequences (shRB) or the negative control vector (shNC) suspended in $100 \mu \mathrm{L}$ cold PBS were inoculated subcutaneously into the flank of the nude mice. Once palpable tumors had developed, the mice were treated with PBS or 5-FU, $20 \mathrm{mg} / \mathrm{kg}$ twice per week. Tumor volumes and body weight were recorded every three days. The tumor volume was calculated as: Volume $=\pi \times$ Length $\times$ Width $\times$ High / 6. All mice were sacrificed 3 weeks after the injection, and the tumors were excised for weight and volume measurement. For the lung metastatic model, approximately $1 \times 10^{6} \mathrm{Hep} 3 \mathrm{~B}$ cells in $100 \mu \mathrm{L}$ PBS were injected into the tail veins of the nude mice. Three days later, the mice were treated with PBS or 5-FU, 20 mg/ $\mathrm{kg}$ twice per week. All mice were sacrificed 8 weeks after the injection, and the lungs were harvested. The metastatic nodules in each lung were counted. The tumors were excised from sacrificed mice, embedded in paraffin and sectioned.

\section{Confocal immunofluorescence}

For confocal microscopy analysis, cells grown on chamber slides were washed with PBS, fixed with paraformaldehyde and permeabilized with pre-cooled methyl alcohol for $15 \mathrm{~min}$ at $-20^{\circ} \mathrm{C}$. The samples were then pretreated with 10\% bovine serum albumin (BSA) in PBS for $30 \mathrm{~min}$, and specific antibodies were added and incubated overnight at $4^{\circ} \mathrm{C}$. Following five $5 \mathrm{~min}$ washes with PBS, secondary antibodies were added and incubated for another hour. After five additional 5 min washes, samples were examined using the confocal microscope.

\section{Immunohistochemistry staining}

Mouse xenograft tumor tissues were analyzed by IHC staining. Briefly, after deparaffinizing, blocking and antigen retrieval, the tumor sections were incubated with primary antibodies at $4^{\circ} \mathrm{C}$ overnight in a humidified chamber. After washing, tumor sections were incubated with horseradish peroxidase-conjugated anti-goat antibodies for $30 \mathrm{~min}$ at room temperature, and colors were developed with 3, 5-diaminobenzidine (DAB) substrate followed by Mayer's hematoxylin counterstaining.

\section{In situ TUNEL assay}

To observe the apoptotic cells in tumor tissues, in situ TUNEL (Keygen Bio-Technology Development Co., Ltd. Nanjing, China) assay was performed on the pathological sections. Briefly, the sections were prepared and deparaffinized in xylene, hydrated through graded alcohols and water, and treated with protease K. Terminal deoxynucleotidyl transferase (TdT) and dUTP-biotin were added and incubated for 1 hour at $37^{\circ} \mathrm{C}$. After washing with PBS, the sections were incubated with streptavidin-HRP and developed with DAB for $10 \mathrm{~min}$.

\section{Statistical analysis}

Results are presented as mean \pm SE of at least three independent experiments. Differences between the mean values of the two subgroups were evaluated using Student's t-test. As for comparison among more than two groups, one-way ANOVA were used. $P<0.05$ was considered to be statistically significant in this study. SPSS 13.0 software was used for all statistical analysis.

\section{Results}

RBFOX3 knockdown suppressed HCC cell growth

To explore the potential function of RBFOX3 in hepatocellular carcinoma, we first evaluated the expression of RBFOX3 in HCC cell lines. We found that the expression of KARGER 
RBFOX3 was higher in human HCC cells (SNU-449, Hep3B, Bel-7402, SNU-387, and HepG2) than in human immortalized hepatic cell line (MIHA) at the protein levels, and Hep3B and HepG2 had higher RBFOX3 expression than the other three HCC cells (Fig. 1A). We next analyzed the expression of RBFOX3 in seven pairs of HCC and paratumor tissues, and found that the protein level of RBFOX3 was significantly higher in HCC tissues than the adjacent non-tumor liver tissues (Fig. 1B). To further examine the biological effects of RBFOX3 on HCC cell growth, we knocked down RBFOX3 and analyzed cell viability by MTS assay and colony formation assay. The results showed that RBFOX3 knockdown (si-RB-1 and si-RB-2) caused dramatic inhibition of cell proliferation (Fig. 1C) and colony formation (Fig. 1D) in HCC cells by comparison with the non-specific siRNA control (si-NC). Moreover, we found that cells in the control group had filopodia and formed a cell layer, whereas RBFOX3 knockdown markedly reduced cell-to-cell contact and filopodia formation (Fig. 1C).

\section{RBFOX3 regulated the sensitivity of HCC to 5-FU through inhibiting the PI3K and AKT} pathway

5 -FU is a frequently used chemotherapeutic drug for the treatment of HCC. However, its dosage is limited by its detrimental side effects. We reasoned that RBFOX3 knockdown might enhance the sensitivity of HCC cells to 5-FU. To test this hypothesis, we first established HepG2 and Hep3B stable cell lines encoding RBFOX3-specific short hairpin RNA sequences (shRB-1 and shRB-2) or the negative control (shNC). We found that the density of RBFOX3knockdown cells treated with 5-FU was significantly decreased compared to the control treated with 5-FU alone in both Hep3B and HepG2 cells (Fig. 2A). We also found that 5-FU inhibited cell viability and colony formation in dose dependent manners, and its inhibitory effect on RBFOX3-knockdown cells was stronger than the control cells (Fig. 2B, left panel and Fig. E). In Hep3B cells, the IC50 values of 5-FU for 72 hours for shNC, shRB-1 and shRB-2 were $50.33 \pm 6.36 \mu \mathrm{mol} / \mathrm{L}, 28.33 \pm 6.36 \mu \mathrm{mol} / \mathrm{L}$ and $26.67 \pm 2.70 \mu \mathrm{mol} / \mathrm{L}$, while the IC50 values for shNC, shRB-1, and shRB-2 in HepG2 cells were 37.00 $\pm 1.91 \mu \mathrm{mol} / \mathrm{L}, 21.33 \pm 2.12 \mu \mathrm{mol} / \mathrm{L}$ and $18.67 \pm 3.54 \mu \mathrm{mol} / \mathrm{L}$ (Fig. $2 \mathrm{~B}$, right panel). Furthermore, we found that 5 -FU inhibited cell viability in time dependent manners, and its inhibitory effect on RBFOX3-knockdown cells was stronger than the control cells (Fig. 2C). We also found its inhibitory effect on RBFOX3knockdown cells was stronger than the control cells in colony formation (Fig. 2F). However, the inhibitory effect of 5-FU on RBFOX3 overexpressing cells was weaker than the control cells in cell viability and colony formation (Fig. 2D and 2G). This indicated that RBFOX3 could regulate the sensitivity of HCC cells to 5-FU.

To elucidate the molecular mechanisms by which RBFOX3 regulated the sensitivity of HCC cells to 5-FU, we analyzed the effect of 5-FU and RBFOX3 on the pivotal proteins in the PI3K/AKT pathway, a crucial pathway associated with tumorigenesis of various human cancers. The results showed that 5-FU alone increased the phosphorylation of PI3K, AKT, and ERK proteins in a dose dependent manner in both Hep3B and HepG2 cells. However, RBFOX3 knockdown in both Hep3B and HepG2 cells dramatically suppressed the phosphorylation of PI3K, AKT, and ERK proteins, and the suppression persisted in the presence of 5-FU (Fig. 2H). Therefore, RBFOX3 knockdown suppressed PI3K-AKT signal activated by 5-FU, resulting in the reduced effective concentration of 5-FU. Our study above suggested that RBFOX3 regulated the sensitivity of HCC cells to 5-FU through the PI3K/AKT pathway.

RBFOX3 regulated 5-FU-induced apoptosis through the cytochrome-c/caspase signaling pathway

Apoptosis is a process of programmed cell death which also influenced cell viability, and most anticancer drugs function primarily to induce apoptosis. We hypothesized that RBFOX3 knockdown potentiated the cytotoxicity of 5-FU via the apoptosis signaling pathway. To test this hypothesis, we measured apoptosis of HCC cells by flow cytometry. As is showed in Fig. 3A, RBFOX3 knockdown potentiated 5-FU-induced apoptosis compared to 5-FU treatment alone. Inversely, we found that overexpression of RBFOX3 decreased the number of apoptotic cells induced by 5-FU in Hep3B and HepG2 cells (Fig. 3B). To elucidate the 


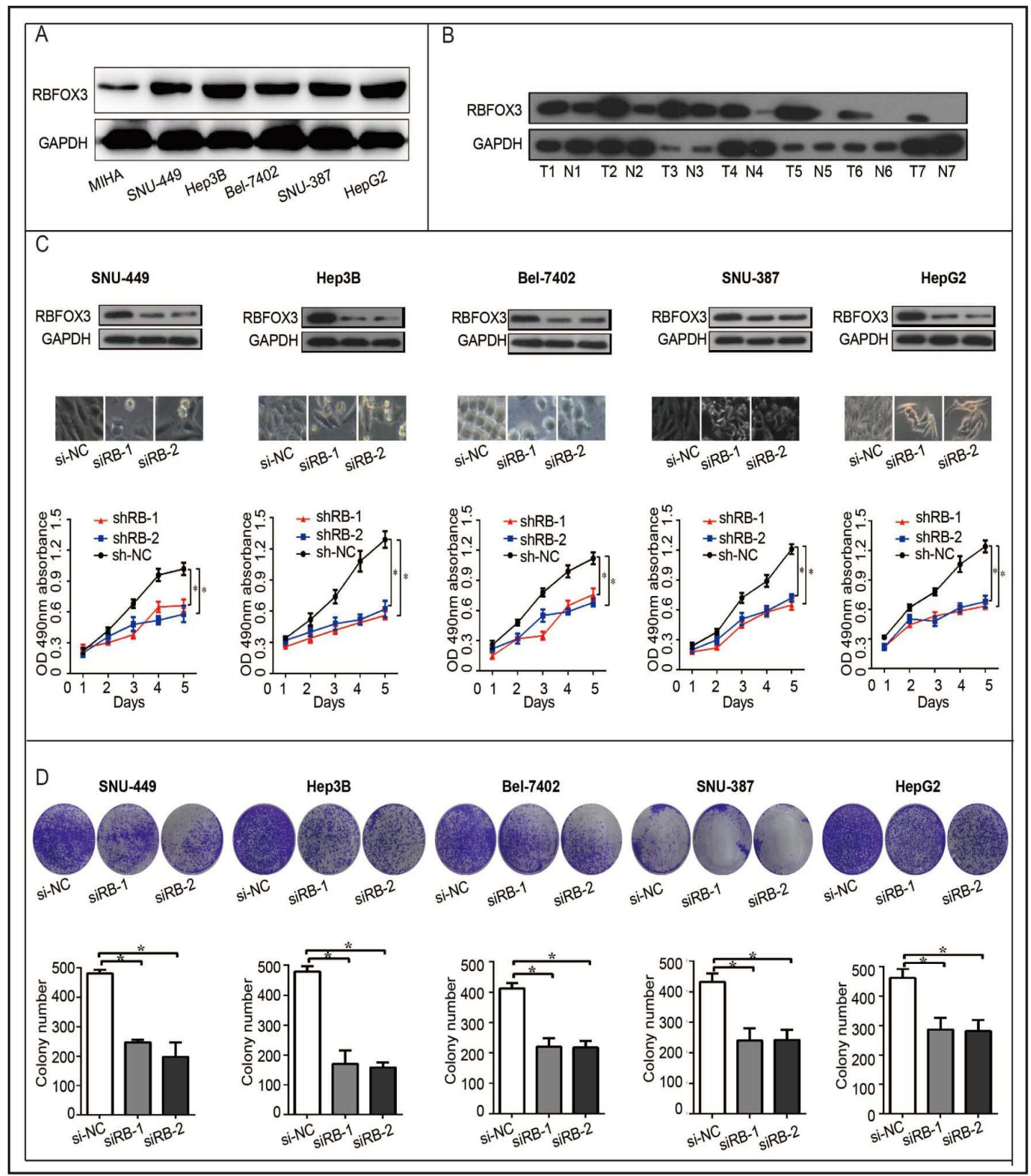

Fig. 1. RBFOX3 knockdown suppressed HCC cell growth. (A) The expression of RBFOX3 in HCC cell lines and human hepatic cell line (MIHA) were analyzed by Western blot. (B) RBFOX3 expression was analyzed by Western blot in HCC samples and adjacent non-tumor liver tissues (n=7), T, tumor tissue; N, adjacent non-tumor tissue. (C) The HCC cells were treated with RBFOX3-specific siRNAs (50 nM) for 72 hours, and the expression of RBFOX3 was detected by Western blot (upper panel). Cell density and morphology were observed with a microscope (middle panel), and cell viability was analyzed by MTS assay (lower panel). (D) HCC cells were treated with RBFOX3-specific siRNA (50 nM) for 14 days. Representative images (upper panel) and quantification (lower panel) of colony formation in Hep3B and HepG2 are shown. Data are presented as mean \pm S.E. $* \mathrm{p}<0.05$.

potential molecular mechanisms, we first performed immunofluorescence (IF) to monitor the subcellular localization of cytochrome c, an upstream molecule of the caspase cascadedependent apoptotic signaling pathway. Fig. 3C showed that treatment with 5-FU triggered the release of cytochrome c (Cyto C) from mitochondria to cytoplasm, which was further

\section{KARGER}




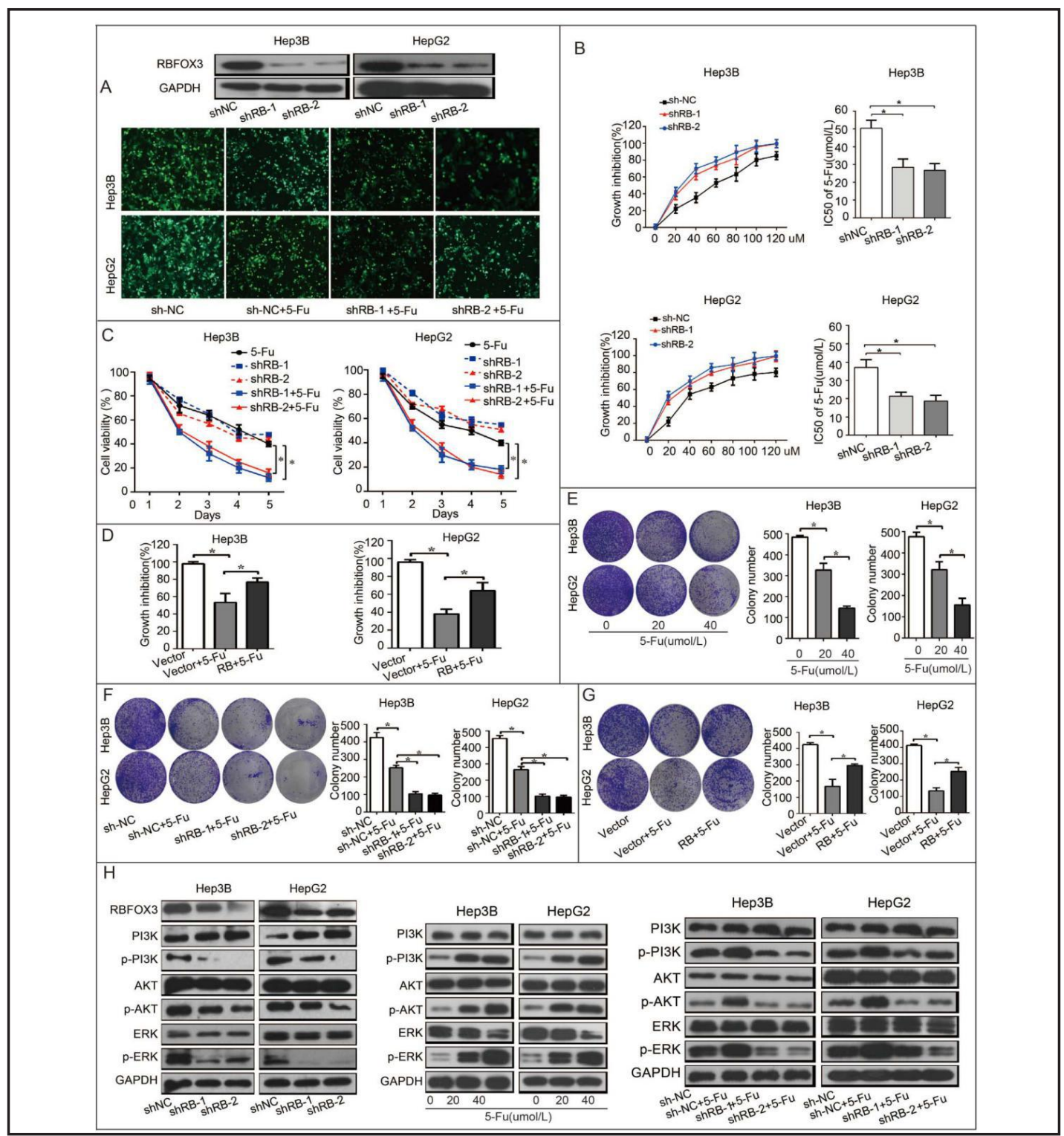

Fig. 2. RBFOX3 regulated the sensitivity of HCC to 5-FU through inhibiting the PI3K and AKT pathway. (A) RBFOX3 knockdown and negative control stable cell lines were constructed by lentivirus-mediated shRNA in Hep3B and HepG2. The efficiency of RBFOX3 knockdown was tested by Western blot (upper panel). Density of the stable cell lines (green fluorescence) was captured by a fluorescence microscope (lower panel). (B) RBFOX3 knockdown or negative control stable Hep3B and HepG2 cells were treated with 5-FU at the indicated doses. At 72 hours after treatment, the cell viability was determined by a MTS assay. IC50 of the stable cell lines exposed to 5-FU was measured. (C) Hep3B and HepG2 cells were transfected with RBFOX3 shRNA (shRB-1 and shRB-2), cell viability was measured by the MTS assay when treated with or without 5-FU at the indicated time. (D) Hep3B and HepG2 cells were treated with 5-FU and/or RBFOX3 overexpression vector. At 72 hours after treatment, the cell viability was determined by a MTS assay. (E) Representative images and quantification of colony formation of Hep3B and HepG2 cells cultured with 5-FU at indicated concentrations for 14 days. (F) Representative images and quantification of colony formation in RBFOX3 knockdown or negative control stable cell lines treated with or without 5-FU for 14 days. (G) Hep3B and HepG2 cells were treated with 5-FU and/or RBFOX3 overexpression vector. At 14 days after treatment, the colony formation was analyzed and calculated. $(\mathrm{H})$ Western blot was used to detect the expression of PI3K, p-PI3K, AKT, p-AKT, ERK, and p-ERK in stable cell lines treated with or without 5-FU. GAPDH was used as a loading control. Data are presented as mean \pm S.E. ${ }^{*} \mathrm{p}<0.05$. 


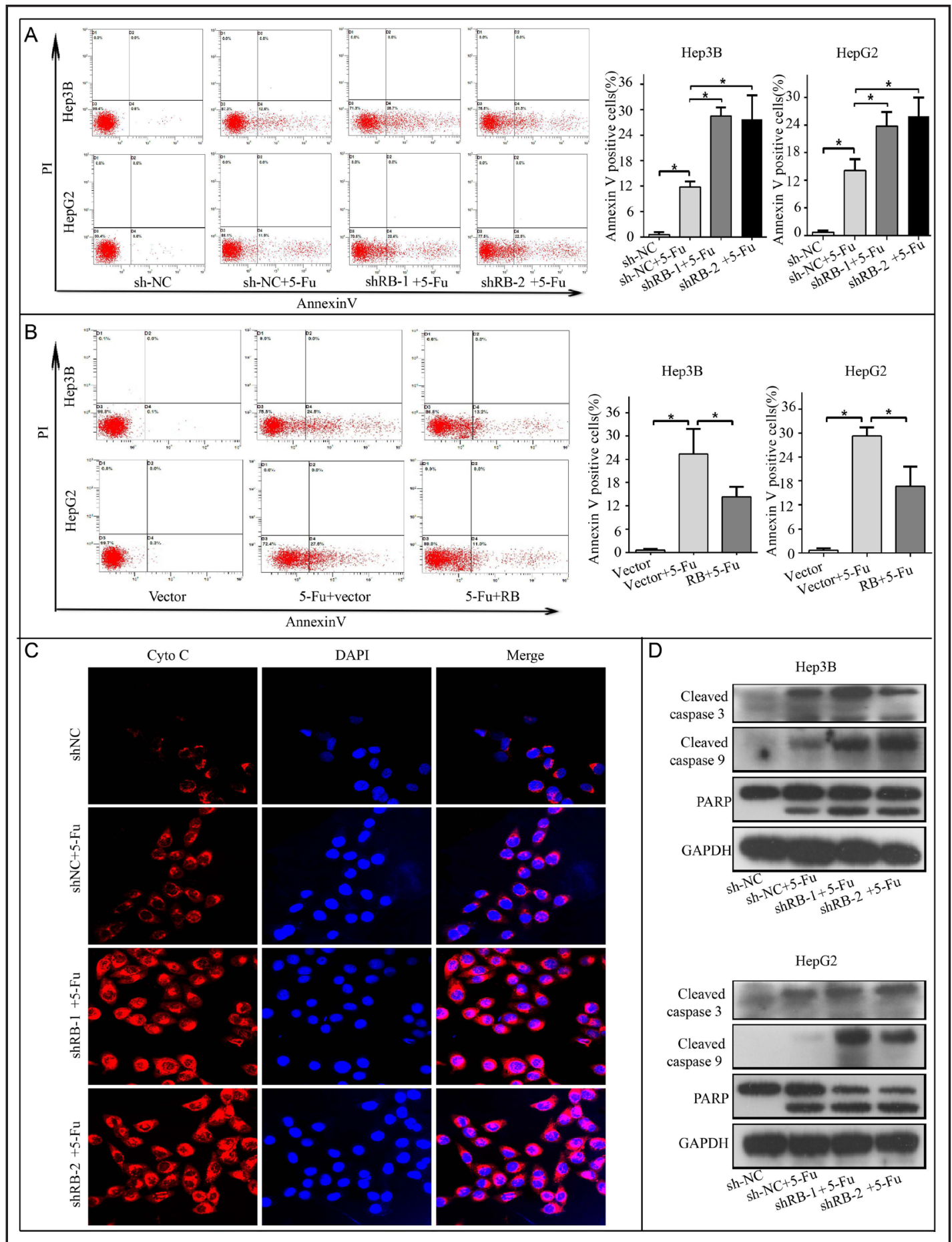

Fig. 3. RBFOX3 regulated 5-FU-induced apoptosis through the cytochrome-c/caspase signaling pathway. (A) Representative images (left panel) and quantification (right panel) of Annexin V/PI assay in RBFOX3 knockdown or negative control stable cell line treated with or without 5-FU for 48 hours. (B) Representative images (left panel) and quantification (right panel) of Annexin V/PI assay in RBFOX3 overexpression or negative control stable cell line treated with or without 5-FU for 48 hours. At 48 hours after treatment, the apoptosis was determined by a FACS analysis.. (C) The release of cytochrome c (cyto-C) was monitored by immunofluorescence analysis in Hep3B stable cell line treated with or without 5-FU. (D) The levels of the cleaved caspase-3, caspase- 9 and PARP proteins were analyzed by Western blot in Hep3B stable cell line treated with or without 5-FU. Data are presented as mean \pm S.E. * $p<0.05$. 


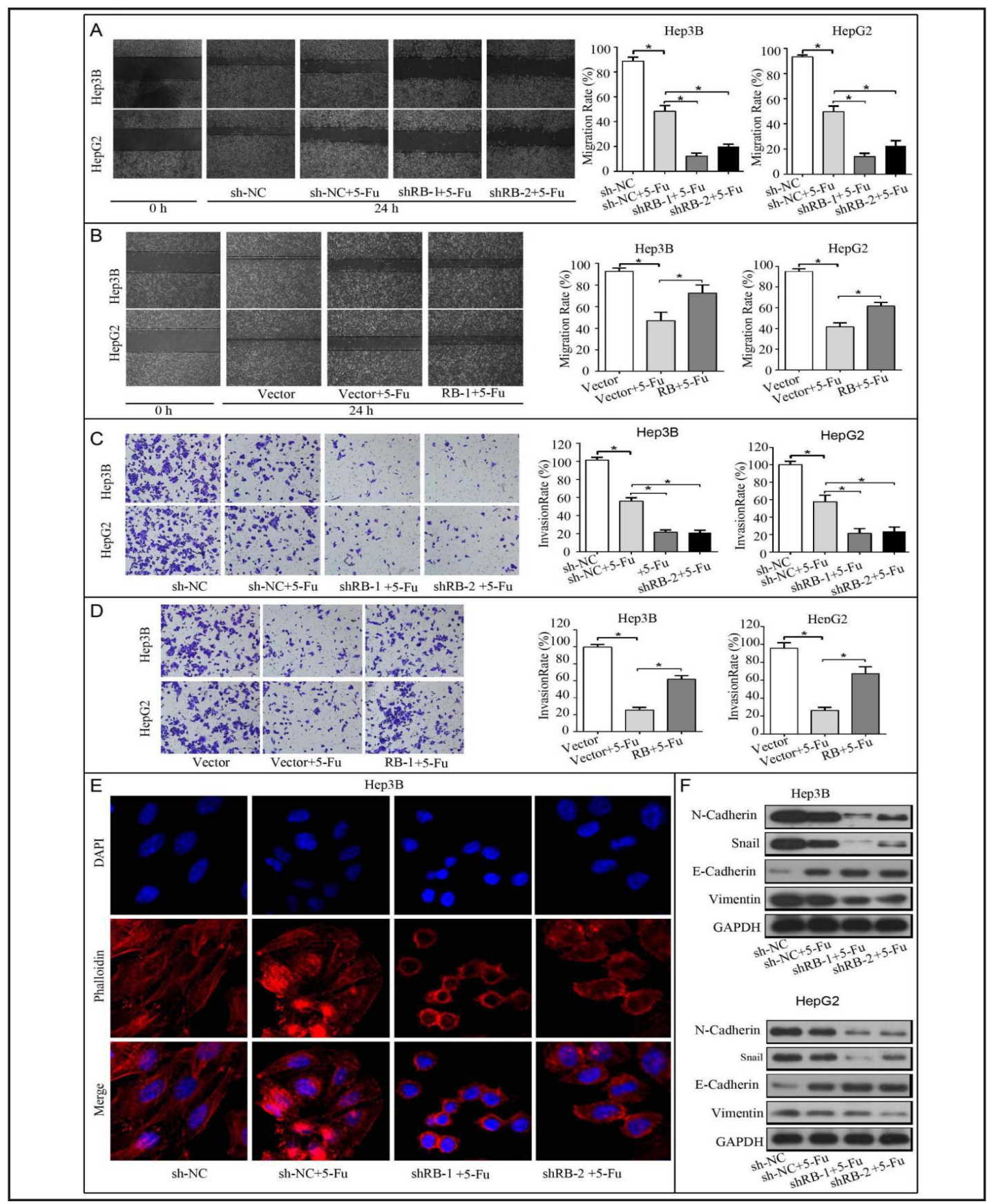

Fig. 4. RBFOX3 regulated 5-FU-mediated inhibition of cell migration and invasion through epithelial-mesenchymal transition (EMT) in HCC cells . (A) Hep3B and HepG2 cells were transfected with RBFOX3 shRNA (shRB-1 and shRB-2) and treated with or without 5-FU. After 24 hours, cell migration was analyzed by a wound-healing assay. (B) Hep3B and HepG2 cells were transfected with RBFOX3 overexpression vector and treated with or without 5-FU. After 24 hours, cell migration was analyzed by a wound-healing assay. (C) Hep3B and HepG2 cells were transfected with RBFOX3 shRNA (shRB-1 and shRB-2) and treated with or without 5-FU. At 24 hours after treatment, cell invasion was analyzed by transwell assay. (D) Hep3B and HepG2 cells were transfected with RBFOX3 overexpression vector and treated with or without 5-FU. After 24 hours, cell invasion was analyzed by transwell assay. (E) Cytoskeleton was analyzed by immunofluorescence assay for phalloidin (red) with DAPI counterstaining (blue) in Hep3B cells. (F) The EMT related protein molecules were analyzed by western blot in Hep3B and HepG2 cells. Data are presented as mean \pm S.E. $* \mathrm{p}<0.05$. 
enhanced in RBFOX3-knockdown cells. We next detected by western blot the downstream key pro-apoptotic proteins of the caspase cascade-dependent apoptotic signaling pathway such as caspase-3, caspase-9, and PARP. We found that 5-FU increased the levels of the cleaved pro-apoptotic proteins, and RBFOX3 knockdown combined with 5-FU further elevated the levels of cleaved PARP, cleaved caspase- 3 and cleaved caspase-9 (Fig. 3D). Thus, these results indicated that RBFOX3 regulated the 5-FU-induced apoptosis through the cytochrome-c/ caspase signaling pathway.

RBFOX3 regulated 5-FU-mediated inhibition of cell migration and invasion through epithelial-mesenchymal transition (EMT) in HCC cells

Distant metastasis is a common cause of HCC related death, and as a result, agents inhibiting metastasis are potential drugs for cancer treatment. To investigate whether RBFOX3 knockdown combined with 5-FU exerted synergistic inhibition on cell migration and invasion, we performed wound healing assay and transwell invasion assay. As is showed in Fig. 4A, RBFOX3 knockdown combined with 5-FU inhibited cell migration more significantly than 5 -FU alone. However, the inhibition of migration mediated by 5-FU was reversed by RBFOX3 overexpression (Fig. 4B). Transwell invasion assay also showed RBFOX3 knockdown combined with 5-FU inhibited cell invasion more significantly than 5-FU alone (Fig. 4C), and the inhibition of invasion mediated by 5-FU could be reversed by RBFOX3 overexpression (Fig. 4D) . Moreover, as epithelial-to-mesenchymal transition (EMT) is crucial for metastasis, we examined the cytoskeleton by IF assay and the expression of proteins related to EMT (N-cadherin, snail and vimentin) by western blot. The results showed that the number of spike-like flopodia at the edges of the cells treated with 5-FU was decreased, and the decrease was more prominent in RBFOX3 cells treated with 5-FU (Fig. 4C). Also, the expressions of the EMT related proteins $\mathrm{N}$-cadherin, snail and vimentin were downregulated while E-cadherin was up-regulated in cells treated by $5-\mathrm{FU}$, which were more significant in RBFOX3-knockdown cells (Fig. 4D). N-cadherin is a cell adhesion molecule that regulates cell-cell adhesion and modulates cell migration during EMT [19], while vimentin is potentially a predictor of EMT behavior [20]. In addition, snail is a transcription factor that represses E-cadherin and mediates EMT [21-23], whereas downregulation of E-cadherin is associated with EMT during embryonic development, a process also exploited by invasive cancer cells [24]. Therefore, these results indicated that RBFOX3 regulated 5-FU-mediated inhibition of cell migration and invasion through EMT in HCC cells.

\section{RBFOX3 regulated 5-FU-mediated inhibition of HCC growth and metastasis in vivo}

To investigate whether RBFOX3 regulated 5-FU-mediated HCC growth in vivo, we utilized a mouse subcutaneous xenograft model. Fifteen BALB/c nude mice were randomly assigned into three groups and inoculated in the flank with Hep3B cells $\left(3 \times 10^{6} /\right.$ mouse $)$ with RBFOX3-specific or control shRNA. The RBFOX3-knockdown group and one control group received 5-FU treatment for three weeks ( $20 \mathrm{mg} / \mathrm{kg}$, twice per week), while the other control group received PBS. As is shown in Fig. 5A, the tumor volume and tumor weight were decreased in the group treated with 5-FU alone when compared to the PBS control group, and were further decreased in the RBFOX3-knockdown combined with 5-FU treatment group. We next analyzed the effect of RBFOX3 knockdown and 5-FU on apoptosis in tumor tissues by TUNEL assay. As is shown in Fig. 5B, the level of apoptosis in the control group was negligible, whereas it became significant in the 5-FU treated group, and even more prominent in the RBFOX3-knockdown with 5-FU treatment group. To further investigate whether RBFOX3 regulated 5-FU-mediated inhibition of tumor metastasis in vivo, the lung metastatic model was established. We found that treatment of 5-FU decreased the number of metastatic nodules compared with the control group, and the number of metastatic nodules was further decreased in the RBFOX3-knockdown with 5-FU treatment group (Fig. 5C). Immunohistochemical staining of the excised tumor sections further revealed that consistent with the in vitro results, RBFOX3 knockdown suppressed the elevation of p-PI3K and p-AKT caused by $5-\mathrm{FU}$, and the expression of $\mathrm{N}$-cadherin, snail and vimentin were significantly 


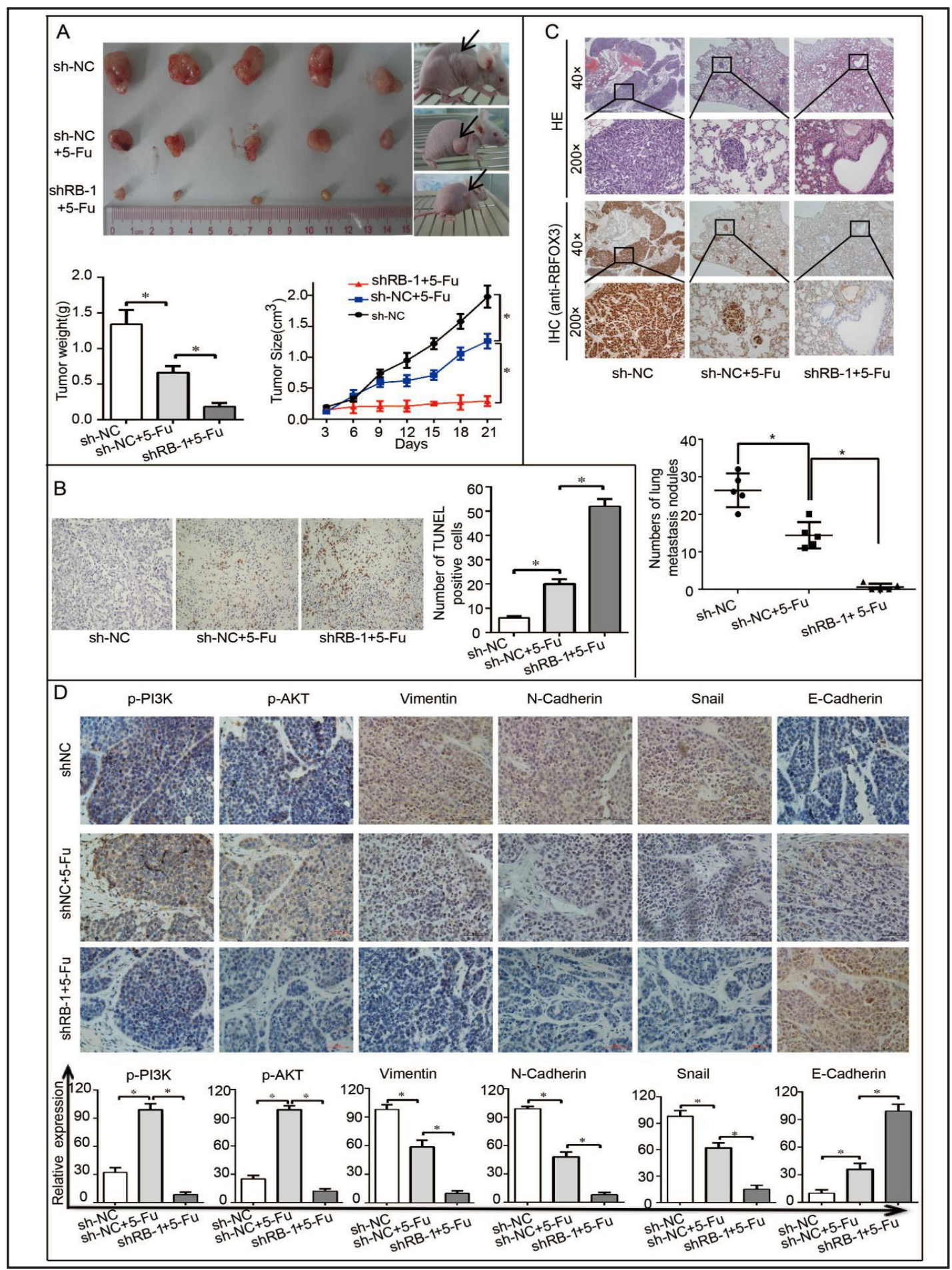

Fig. 5. RBFOX3 regulated 5-FU-mediated inhibition of HCC growth and metastasis in vivo. (A) The nude mice bearing xenografts of HCC cells with or without RBFOX3 knockdown were intratumorally treated with or without 5-FU. The tumor volume and tumor weight were measured after treatment. (B) Representative images of TUNEL staining in tumor sections from each group, with quantification performed by counting the TUNEL positive cells from each group. (C) Representative hematein-eosin (H\&E) and immunohistochemical (RBFOX3) staining of the metastatic lung nodules and quantification results. (D) Representative immunohistochemical staining and quantification of p-PI3K, p-AKT, Vimentin, N-Cadherin, Snail and E-Cadherin from tumour sections. Data are presented as mean \pm S.E. * $\mathrm{p}<0.05$. 
decreased while E-cadherin was significantly increased in the RBFOX3-knockdown with 5-FU treatment group when compared with the group treated with 5-FU alone (Fig. 5D). These data further indicate that RBFOX3 knockdown synergized with 5-FU to regulate HCC tumor growth, EMT and apoptosis in vivo.

\section{Discussion}

Over the past decade, HCC is one of the few cancers that have a rise in incidence. Though the treatment of HCC has made some progress, much more effort is needed to improve the treatment effect. As a pyrimidine analog, 5-FU is used extensively for the clinical treatment of various types of solid tumors, including breast [25], colorectal [26], HCC [27], stomach and gullet cancers [28]. However, 5-FU resistance is a malignant phenotype, and its dosage was limited because of its side effects including gastrointestinal reaction and palmar plantar erythroderma [29]. Therefore, it is necessary to find new therapeutic targets that can synergize with 5 -FU in cancer treatment.

The RNA-binding protein RBFOX3 is a well-known splicing regulator used as a marker for post-mitotic neurons in various vertebrate species. Recent genetic studies have implicated that RBFOX3 is involved in numerous neurological dysfunctions in humans, such as the development of autistic features, epilepsy, cognitive impairments, and neurodevelopmental delay. Although previous studies reported RBFOX3 was exclusively expressed in post-mitotic neurons of the central nervous system, recent studies indicated that RBFOX3 is also expressed in some non-neuronal tissues [30,31]. Another study showed that RBFOX3 expression was inhibited during TGF- $\beta$-induced epithelial-mesenchymal transition (EMT) in lung cancer [32]. Nevertheless, the cellular function of RBFOX3 in tumorigenesis remains unclear. Here, we report that RBFOX3 was highly expressed in HCC cell lines and tissues, and RBFOX3 knockdown inhibited HCC cell growth and colony formation. What's more, the sensitivity of HCC cells to 5-FU was enhanced with RBFOX3 knockdown, as 5-FU had a stronger growth inhibition effect on the RBFOX3-knockdown cells than the control cells. We further found that RBFOX3 knockdown enhanced the growth inhibition by 5-FU through targeting the PI3K/AKT pathway. As one of the most important pathways participating in cells growth, the PI3K/AKT pathway is generally inactivated with treatment of most anticancer drugs. However, chemotherapy-induced activation of cell survival pathways has been observed [33], which may lead to drug resistance. For example, drugs like paclitaxel, pemetrexed, and docetaxel have been reported to activate cell survival pathways such as PI3K/AKT or EGFR pathway [34-36]. Consistent with the results from esophageal squamous cell carcinoma cells exposed to 5-FU [37], our study also found that 5-FU inhibited HCC cell growth with increased phosphorylation of PI3K, AKT and ERK proteins, indicating that 5-FU suppressed cell growth via other pathways. Since activation of the PI3K/AKT pathway may partly offset the inhibition of cell growth, combinatorial treatment with 5-FU and another agent that inactivates the PI3K/AKT pathway will improve the anticancer effect of 5-FU. Consistently, our study demonstrated that RBFOX3 knockdown synergized with 5-FU by inactivating the PI3K/AKT pathway.

We also tried to identify other potential mechanisms by which RBFOX3 knockdown increased the sensitivity to 5-FU of HCC cells. As most anticancer drugs function primarily to induce apoptosis [38], and emerging data have indicated that 5-FU alone could induce apoptosis in gastric [39], colorectal [40] and thymic cells [41], we examined whether RBFOX3 knockdown increased 5-FU-induced apoptosis. Our results showed that RBFOX3 knockdown increased the levels of cleaved PARP, caspase-3 and caspase-9 in HCC cells, and synergized with 5-FU in inducing apoptosis by increasing the release of cytochrome-c and the levels of the cleaved pro-apoptotic proteins, indicating that RBFOX3 knockdown enhanced 5-FUinduced apoptosis by activating the cytochrome-c/caspase signaling pathway.

The prognosis of HCC patients remains unsatisfactory due to a high incidence of metastasis, which contributes to around $90 \%$ of cancer-related mortality $[42,43]$. Therefore, 


\section{Cellular Physiology Cell Physiol Biochem 2018:46:1365-1380 \begin{tabular}{ll|l} 
DOI: 10.1159/000489153 & $\begin{array}{l}\text { O 2018 The Author(s). Published by S. Karger AG, Basel } \\
\text { www.karger.com/cpb }\end{array}$ \\
\hline
\end{tabular}

Fig. 6. The schematic diagram illustrating the molecular mechanisms by which RBFOX3 knockdown enhanced 5-FU-mediated inhibition of growth and metastasis in HCC. (Left panel) RBFOX3 knockdown enhanced 5-FU-mediated inhibition of cell migration and invasion by inhibiting EMT in HCC cells. (Middle panel) RBFOX3 knockdown dramatically suppressed the phosphorylation of PI3K, AKT, and ERK proteins which were elevated by 5 -Fu. (Right panel) RBFOX3 knockdown potentiated 5-FU-induced apoptosis by activating cytochromec/caspase signaling pathway.

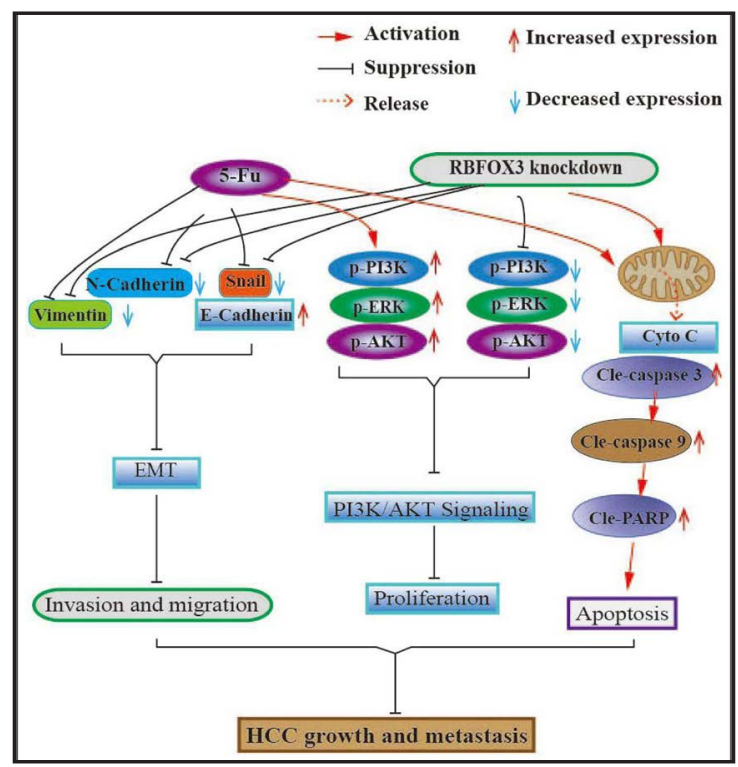

it is necessary to identify prognostic markers and treatment targets for metastatic HCC. Results from wound healing and transwell invasion assay suggested that RBFOX3 knockdown combined with 5-FU inhibited cell migration and invasion more significantly than treatment with 5-FU alone. As epithelial-mesenchyme transition (EMT) is known to be a central mechanism responsible for invasiveness and metastasis of various cancers [44], and previous studies reported that RBFOX3 and 5-FU were involved in EMT [45, 46], we explored whether RBFOX3 knockdown enhanced 5-FU-mediated inhibition of cell migration and invasion through EMT. We found fewer spike-like flopodia, a characteristic of invasive cancer cells, in RBFOX3-knockdown cells treated with 5-FU than the 5-FU-treated control cells. Consistently, EMT related proteins N-cadherin, snail and vimentin were further down-regulated with E-cadherin further up-regulated in RBFOX3-knockdown cells treated with 5-FU compared with control cells treated with 5-FU alone. Based on these findings, we believe that RBFOX3 knockdown enhanced 5-FU-mediated inhibition of cell migration and invasion through inhibiting EMT.

\section{Conclusion}

Taken together, findings from the present study indicate that RBFOX3 regulated 5-FUmediated cytotoxicity via the PI3K/AKT, EMT and cytochrome-c/caspase pathway in HCC both in vitro and in vivo (Fig. 6).

\section{Abbreviations}

HCC (hepatocellular carcinoma); 5-FU (5-fluorouracil); EMT (epithelial-mesenchymal transition); PSF (protein-associated splicing factor); IF (immunofluorescence); CytoC (cytochrome c).

\section{Acknowledgements}

This work was supported by the funds from the National Natural Science Foundation of China (81772925, 81472178, 81401905, 81702761), Natural Science Foundation of Guangdong Province (2016A03031100; 2015A030313018), Guangdong Esophageal Cancer Institute (2015A09), Guangzhou Science Technology and Innovation Commission (201607020038), and the State “973 Program” of China (2014CB542005). 


\section{Cellular Physiology Cell Physiol Biochem 2018;46:1365-1380 \begin{tabular}{ll|l} 
and Biochemistry Published online: April 23, 2018 & $\begin{array}{l}\text { C } 2018 \text { The Author(s). Published by S. Karger AG, Basel } \\
\text { www.karger.com/cpb }\end{array}$ \\
\hline
\end{tabular}}

Liu et al.: RBFOX3 Regulates the Chemosensitivity

\section{Disclosure Statement}

No conflict of interests exists.

\section{References}

$>1$ Gao Y, Ruan B, Liu W, Wang J, Yang X, Zhang Z, Li X, Duan J, Zhang F, Ding R, Tao K, Dou K: Knockdown of CD44 inhibits the invasion and metastasis of hepatocellular carcinoma both in vitro and in vivo by reversing epithelial-mesenchymal transition. Oncotarget 2015;6:7828-7837.

-2 Xu J, Li X, Yang H, Chang R, Kong C, Yang L: SIN1 promotes invasion and metastasis of hepatocellular carcinoma by facilitating epithelial-mesenchymal transition. Cancer 2013;119:2247-2257.

-3 Singal AG, Pillai A, Tiro J: Early detection, curative treatment, and survival rates for hepatocellular carcinoma surveillance in patients with cirrhosis: a meta-analysis. PLoS Med 2014;11:e1001624.

-4 Jiao HK, Xu Y, Li J, Wang W, Mei Z, Long XD, Chen GQ: Prognostic significance of Cbx4 expression and its beneficial effect for transarterial chemoembolization in hepatocellular carcinoma. Cell Death Dis 2015;6:e1689.

5 Koniaris LG, Levi DM, Pedroso FE, Franceschi D, Tzakis AG, Santamaria-Barria JA, Tang J, Anderson M, Misra S, Solomon NL, Jin X, DiPasco PJ, Byrne MM, Zimmers TA: Is surgical resection superior to transplantation in the treatment of hepatocellular carcinoma? Ann Surg 2011;254:527-537.

6 Hernandez-Gea V, Toffanin S, Friedman SL, Llovet JM: Role of the microenvironment in the pathogenesis and treatment of hepatocellular carcinoma. Gastroenterology 2013;144:512-527.

-7 Zhang PF, Li KS, Shen YH, Gao PT, Dong ZR, Cai JB, Zhang C, Huang XY, Tian MX, Hu ZQ, Gao DM, Fan J, Ke AW, Shi GM: Galectin-1 induces hepatocellular carcinoma EMT and sorafenib resistance by activating FAK/ PI3K/AKT signaling. Cell Death Dis 2016;7:e2201.

$\checkmark 8$ Vilarinho S, Calvisi DF: New advances in precision medicine for hepatocellular carcinoma recurrence prediction and treatment. Hepatology 2014;60:1812-1814.

-9 Sakabe T, Tsuchiya H, Kanki K, Azumi J, Gonda K, Mizuta Y, Yamada D, Wada H, Shomori K, Nagano H, Shiota G: Identification of the genes chemosensitizing hepatocellular carcinoma cells to interferon-alpha/5fluorouracil and their clinical significance. PloS one 2013;8:e56197.

10 Wang W, Liu WB, Huang da B, Jia W, Ji CS, Hu B: Targeting PCDH20 gene by microRNA-122 confers 5-FU resistance in hepatic carcinoma. Am J Cancer Res 2016;6:1681-1694.

11 Gao S, Zhang PJ, Guo JH, Chen H, Xu HF, Liu P, Yang RJ, Zhu X: Chemoembolization alone vs combined chemoembolization and hepatic arterial infusion chemotherapy in inoperable hepatocellular carcinoma patients. World J Gastroenterol 2015;21:10443-10452.

12 Li X, Yu J, Brock MV, Tao Q, Herman JG, Liang P, Guo M: Epigenetic silencing of BCL6B inactivates p53 signaling and causes human hepatocellular carcinoma cell resist to 5-FU. Oncotarget 2015;6:11547-11560.

13 Hyuga S, Shiraishi M, Hori A, Hyuga M, Hanawa T: Effects of Kampo medicines on MDR-1-mediated multidrug resistance in human hepatocellular carcinoma HuH-7/PTX cells. Biol Pharm Bull 2012;35:17291739.

14 Duan W, Zhang YP, Hou Z, Huang C, Zhu H, Zhang CQ, Yin Q: Novel Insights into NeuN: from Neuronal Marker to Splicing Regulator. Mol Neurobiol 2016;53:1637-1647.

15 Kim KK, Nam J, Mukouyama YS, Kawamoto S: Rbfox3-regulated alternative splicing of Numb promotes neuronal differentiation during development. J Cell Biol 2013;200:443-458.

-16 Mullen RJ, Buck CR, Smith AM: NeuN, a neuronal specific nuclear protein in vertebrates. Development 1992;116:201-211.

-17 Liu T, Li W, Lu W, Chen M, Luo M, Zhang C, Li Y, Qin G, Shi D, Xiao B, Qiu H, Yu W, Kang L, Kang T, Huang W, Yu X, Wu X, Deng W: RBFOX3 Promotes Tumor Growth and Progression via hTERT Signaling and Predicts a Poor Prognosis in Hepatocellular Carcinoma. Theranostics 2017;7:3138-3154.

18 Kim KK, Yang Y, Zhu J, Adelstein RS, Kawamoto S: Rbfox3 controls the biogenesis of a subset of microRNAs. Nat Struct Mol Biol 2014;21:901-910.

19 Zhu K, Dai Z, Pan Q, Wang Z, Yang GH, Yu L, Ding ZB, Shi GM, Ke AW, Yang XR, Tao ZH, Zhao YM, Qin Y, Zeng HY, Tang ZY, Fan J, Zhou J: Metadherin promotes hepatocellular carcinoma metastasis through induction of epithelial-mesenchymal transition. Clin Cancer Res 2011;17:7294-7302. 


\section{Cellular Physiology Cell Physiol Biochem 2018;46:1365-1380 \begin{tabular}{l|l} 
DOI: 10.1159/000489153 & $\begin{array}{l}\text { O } 2018 \text { The Author(s). Published by S. Karger AG, Basel } \\
\text { www.karger.com/cpb }\end{array}$
\end{tabular}}

Liu et al.: RBFOX3 Regulates the Chemosensitivity

20 Raymond WA, Leong AS: Vimentin--a new prognostic parameter in breast carcinoma? J Pathol 1989;158:107-114.

-21 Batlle E, Sancho E, Franci C, Dominguez D, Monfar M, Baulida J, Garcia De Herreros A: The transcription factor snail is a repressor of E-cadherin gene expression in epithelial tumour cells. Nat Cell Biol 2000;2:8489.

22 Barrallo-Gimeno A, Nieto MA: The Snail genes as inducers of cell movement and survival: implications in development and cancer. Development 2005;132:3151-3161.

23 Cano A, Perez-Moreno MA, Rodrigo I, Locascio A, Blanco MJ, del Barrio MG, Portillo F, Nieto MA: The transcription factor snail controls epithelial-mesenchymal transitions by repressing E-cadherin expression. Nat Cell Biol 2000;2:76-83.

24 Huang RY, Guilford P, Thiery JP: Early events in cell adhesion and polarity during epithelial-mesenchymal transition. J Cell Sci 2012;125:4417-4422.

25 Martin M, Pienkowski T, Mackey J, Pawlicki M, Guastalla JP, Weaver C, Tomiak E, Al-Tweigeri T, Chap L, Juhos E, Guevin R, Howell A, Fornander T, Hainsworth J, Coleman R, Vinholes J, Modiano M, Pinter T, Tang SC, Colwell B, Prady C, Provencher L, Walde D, Rodriguez-Lescure A, Hugh J, Loret C, Rupin M, Blitz S, Jacobs P, Murawsky M, Riva A, Vogel C, Breast Cancer International Research Group I: Adjuvant docetaxel for nodepositive breast cancer. N Engl J Med 2005;352:2302-2313.

26 Macdonald JS, Astrow AB: Adjuvant therapy of colon cancer. Semin Oncol 2001;28:30-40.

27 Lin DY, Lin SM, Liaw YF: Non-surgical treatment of hepatocellular carcinoma. J Gastroenterol Hepatol 1997;12:S319-328.

28 Macdonald JS, Smalley SR, Benedetti J, Hundahl SA, Estes NC, Stemmermann GN, Haller DG, Ajani JA, Gunderson LL, Jessup JM, Martenson JA: Chemoradiotherapy after surgery compared with surgery alone for adenocarcinoma of the stomach or gastroesophageal junction. N Engl J Med 2001;345:725-730.

29 Shi L, Wu L, Chen Z, Yang J, Chen X, Yu F, Zheng F, Lin X: MiR-141 Activates Nrf2-Dependent Antioxidant Pathway via Down-Regulating the Expression of Keap1 Conferring the Resistance of Hepatocellular Carcinoma Cells to 5-Fluorouracil. Cell Physiol Biochem 2015;35:2333-2348.

-30 Langenfeld E, Deen M, Zachariah E, Langenfeld J: Small molecule antagonist of the bone morphogenetic protein type I receptors suppresses growth and expression of Id1 and Id3 in lung cancer cells expressing Oct4 or nestin. Mol Cancer 2013;12:129.

31 Shuangshoti S, Mujananon S, Chaipipat M, Keetacheeva K, Shuangshoti S: Expression of neuronal nuclear antigen (NeuN) in epithelial neuroendocrine carcinoma. Appl Immunohistochem Mol Morphol 2005;13:265-267.

32 Kim YE, Kim JO, Park KS, Won M, Kim KE, Kim KK: Transforming Growth Factor-beta-Induced RBFOX3 Inhibition Promotes Epithelial-Mesenchymal Transition of Lung Cancer Cells. Mol Cells 2016;39:625-630.

33 Torres K, Horwitz SB: Mechanisms of Taxol-induced cell death are concentration dependent. Cancer Res 1998;58:3620-3626.

-34 Cheng H, An SJ, Zhang XC, Dong S, Zhang YF, Chen ZH, Chen HJ, Guo AL, Lin QX, Wu YL: In vitro sequencedependent synergism between paclitaxel and gefitinib in human lung cancer cell lines. Cancer Chemother Pharmacol 2011;67:637-646.

-35 Li T, Ling YH, Goldman ID, Perez-Soler R: Schedule-dependent cytotoxic synergism of pemetrexed and erlotinib in human non-small cell lung cancer cells. Clin Cancer Res 2007;13:3413-3422.

-36 Chen B, Zheng J, Zeng Y, Li B, Xie B, Zheng J, Zhou J, Zhang W: Sequence-dependent antiproliferative effects of gefitinib and docetaxel on non-small cell lung cancer (NSCLC) cells and the possible mechanism. PloS one 2014;9:e114074.

37 Lu YX, Chen DL, Wang DS, Chen LZ, Mo HY, Sheng H, Bai L, Wu QN, Yu HE, Xie D, Yun JP, Zeng ZL, Wang F, Ju HQ, Xu RH: Melatonin enhances sensitivity to fluorouracil in oesophageal squamous cell carcinoma through inhibition of Erk and Akt pathway. Cell Death Dis 2016;7:e2432.

38 Brunelle JK, Zhang B: Apoptosis assays for quantifying the bioactivity of anticancer drug products. Drug Resist Updat 2010;13:172-179.

-39 Osaki M, Tatebe S, Goto A, Hayashi H, Oshimura M, Ito H: 5-Fluorouracil (5-FU) induced apoptosis in gastric cancer cell lines: role of the p53 gene. Apoptosis 1997;2:221-226.

40 Cao S, Lu K, Toth K, Slocum HK, Shirasaka T, Rustum YM: Persistent induction of apoptosis and suppression of mitosis as the basis for curative therapy with S-1, an oral 5-fluorouracil prodrug in a colorectal tumor model. Clin Cancer Res 1999;5:267-274. 


\section{Cellular Physiology Cell Physiol Biochem 2018;46:1365-1380 \begin{tabular}{l|l} 
DOI: 10.1159/000489153 & $\begin{array}{l}\text { O 2018 The Author(s). Published by S. Karger AG, Basel } \\
\text { www.karger.com/cpb }\end{array}$ \\
\hline
\end{tabular}}

Liu et al.: RBFOX3 Regulates the Chemosensitivity

41 Eichhorst ST, Muerkoster S, Weigand MA, Krammer PH: The chemotherapeutic drug 5-fluorouracil induces apoptosis in mouse thymocytes in vivo via activation of the CD95(AP0-1/Fas) system. Cancer Res 2001;61:243-248.

42 Kai AK, Chan LK, Lo RC, Lee JM, Wong CC, Wong JC, Ng IO: Down-regulation of TIMP2 by HIF-1alpha/ miR-210/HIF-3alpha regulatory feedback circuit enhances cancer metastasis in hepatocellular carcinoma. Hepatology 2016;64:473-487.

43 Wong CM, Wei L, Law CT, Ho DW, Tsang FH, Au SL, Sze KM, Lee JM, Wong CC, Ng IO: Up-regulation of histone methyltransferase SETDB1 by multiple mechanisms in hepatocellular carcinoma promotes cancer metastasis. Hepatology 2016;63:474-487.

44 Du R, Wu S, Lv X, Fang H, Wu S, Kang J: Overexpression of brachyury contributes to tumor metastasis by inducing epithelial-mesenchymal transition in hepatocellular carcinoma. J Exp Clin Cancer Res 2014;33:105.

45 Arumugam T, Ramachandran V, Fournier KF, Wang H, Marquis L, Abbruzzese JL, Gallick GE, Logsdon CD, McConkey DJ, Choi W: Epithelial to mesenchymal transition contributes to drug resistance in pancreatic cancer. Cancer Res 2009;69:5820-5828.

46 Toden S, Okugawa Y, Jascur T, Wodarz D, Komarova NL, Buhrmann C, Shakibaei M, Boland CR, Goel A: Curcumin mediates chemosensitization to 5-fluorouracil through miRNA-induced suppression of epithelial-to-mesenchymal transition in chemoresistant colorectal cancer. Carcinogenesis 2015;36:355367. 